\title{
Ellipticity in Cosmic Microwave Background as a Tracer of Large-Scale Universe
}

\author{
V.G.Gurzadyan ${ }^{1,2}$, C.L.Bianco ${ }^{2}$, A.L.Kashin ${ }^{1}$, H.Kuloghlian ${ }^{1}$, G.Yegorian ${ }^{1,2}$ \\ 1 Yerevan Physics Institute, Yerevan, Armenia; ${ }^{2}$ ICRANet, ICRA, Di- \\ partimento di Fisica, Universita La Sapienza, Roma, Italy
}

\begin{abstract}
Wilkinson Microwave Anisotropy Probe (WMAP) 3-year data confirm the ellipticity of anisotropies of Cosmic Microwave Background (CMB) maps, found previously for Boomerang and WMAP 1-year high sensitivity maps. The low noise level of the WMAP latter data enable also to show that, the ellipticity is a property not described by the conventional cosmological model fitting the power spectrum of CMB. As a large scale anomaly, the ellipticity characteristics are consistent with the effect of geodesics mixing occurring in hyperbolic Universe. Its relation to other large scale effects, i.e. to suppressed low multipoles, as well as to dark energy if the latter is due to vacuum fluctuations, is then an arising issue.
\end{abstract}

The analysis of high signal to noise ratio $150 \mathrm{GHz}$ Cosmic Microwave Background (CMB) radiation maps obtained in the Boomerang 1998 experiment 1], 2] has recently revealed ellipticity about 2 of the temperature anisotropies [3. Wilkinson Microwave Anisotropy Probe's (WMAP) 1-year data 44 confirmed the results of Boomerang [5]. Initially a signature of ellipticity was found for COBE-DMR maps [6]. The effect was shown to exist at scales both larger and smaller than the horizon scale at the last scattering epoch [7].

There are various unexpected features detected in the CMB sky, such as the quadrupole-octopole correlation, planarity of the octopole, the cold spot, the Axis of Evil, etc. (e.g. [8, 9, 10, 11, 12, 13, 14, 15]), which are being reanalysed by WMAP 3-year data [16, 17, 18].

WMAP 3-year data provide also a possibility to follow the ellipticity effect with higher signal-to-noise ratio, thus checking not only the value of the ellipticity but also the role of the noise in comparison with simulated maps.

Originally, the interest to the ellipticity was motivated by the effect of geodesic mixing which has to exist in hyperbolic spaces due to exponential divergence of close null geodesics in $(3+1)$-space [19]. These properties of hyperbolic spaces are well known both in geometry [20] and in theory of 
hyperbolic dynamical systems [21]. Another descriptor for CMB maps, the Kolmogorov complexity, was suggested in 22].

The algorithms of study of the ellipticties in pixelized CMB maps have been described in previous papers [3, 5] and are reduced, first, to the determination of the anisotropy spots (excursion sets) at given temperature thresholds, and then, to the definition of their semi-axes. Although motivated by the geodesic mixing, the measurement of mean elongation actually implies a more general content, namely, estimation of a Lyapunov exponent of any dynamical system which might be its reason.

WMAP 3-year data not only confirmed the value of the ellipticity detected before in CMB maps, but enabled to draw new conclusions. In previous papers we had mentioned the impossibility of direct tracing of the effect via maps simulated for the power spectrum parameters. The reason is that a possible fit of simulations to real data could not be considered as a proof of the model, since the ellipticity initially was not within the parameters of the model, and hence any other model may fit better. However, now due to low noise level of WMAP 3-year data it appears possible to reveal the practically genuine ellipticity and compare the sequence of data maps of various noise level with simulated maps.

We found that, first, for simulated signal maps (no noise) the ellipticity is increasing for the large spots while for the data maps it is the contrary, decreasing. Second, the contribution of the WMAP 3-year noise in the simulated maps is not only far larger, but is opposite for spots of various size. Namely, for more than 20-pixel spots the noise is increasing the ellipticity, while for more than 50 or 100-pixel spots we see the contrary, a decrease. So, both, by behavior and amplitude, the ellipticity of the simulated maps contradicts the real maps.

This implies that the ellipticity is an effect not included in the cosmological model fitting the power spectrum. Note, that for $\Lambda C D M$ model the Boomerang power spectra simulated maps have ellipticity around 1.8 [3] for the noise level of Boomerang, while for the once popular flat CDM model, with no noise, the mean ellipticity is around 1.4 [23].

The independence of the effect on the scale of the horizon at the last scattering surface shows that it cannot be related to the physical conditions there, and hence, most probably we deal with a large scale effect. Note, nonzero curvature or non-trivial topology models (e.g. [24, 25, 26]) are among the models proposed to explain the low quadrupole.

We used the WMAP 3-year $94 \mathrm{GHz}(3.2 \mathrm{~mm})$ maps, i.e. the same channel as we had used for the 1-year data [5], since they have highest angular resolution (beam size $0.22^{\circ} \mathrm{FWHM}$ ) and are least influenced by the Galactic 
synchrotron radiation.

To study the role of the noise via the real data we produced a sequence of maps of various noise level combining the data from four independent detectors $w_{i}$ into two independent channels $A=\left(w_{1}+w_{2}\right) / 2$ and $B=$ $\left(w_{3}+w_{4}\right) / 2$ in Healpix [27] representation with nside $=512(6.9$ arcmin pixel side), when the pixels with galactic latitude $|b|<20^{\circ}$ were excluded, as usual. The algorithms for the definition of the excursion sets on the sphere, as well as for the definition of the two semi-axes and of ellipticity as of their ratio, are described in in details in [3, 7]. We outline that, 3 types of possible errors have been studied separately. First, the statistical ones appear after avareging over the number of the spots for the given threshold, second, the bias of the algorithms have been thoroughly studied via variation of the input parameters and algorithmic steps, third, the systematic errors have been studied via the sequence of real maps with different noise level.

In Fig. 1 we represent the average ellipticity of the anisotropy spots counting more than 20, 50 and 100 pixels, as a function of the temperature threshold for the $A+B$ map. The error bars in Fig.1 are statistical only, as they are computed from the standard deviation of the ellipticities of all the areas at a given threshold. As we have shown in [3], for areas containing more than several tens of pixels, the algorithm bias does not exceed 0.1 in the ellipticity. The statistics of the spots is given in Fig. 2 and is quite consistent with WMAP 1-year data [5]. Fig. 3 exhibits the frequency dependence of the ellipticity. As compared to $\mathrm{w}$ band, the ellipticity homogeneity is distorted, especially, at $41 \mathrm{GHz}$ (q band), where the Galactic foregroud is stronger (possibly, due to anomalous dust emission with steep fall at 60 $\mathrm{GHz}$ [18, 29]. In Fig. 4 we compare two maps, without $|b|<20^{\circ}$ pixels (as in Fig. 1) and without $|b|<30^{\circ}$, thus showing the absence of any essential role of the Galactic disk. Fig. 5 shows the ellipticity in first and second yearly maps, along with the 3-year $94 \mathrm{GHz}$ one.

The procedure first adopted in [5] to reveal the role of the noise in the ellipticity using not the simulations but the real data, is repeated here. Namely, we obtained new maps by adding the difference data $A-B$, which contain only noise, to different combinations of the $w_{i}$ data, which contain signal and noise. This procedure effectively changes the signal to noise ratio $(\mathrm{S} / \mathrm{N})$ of the map. We analyzed the following maps: $(A+B) / 2 ;\left(A^{\prime}+B\right) / 2$, where $A^{\prime}=\left(w_{1}+A-B+w_{2}\right) / 2 ; w_{1} ; w_{1}+A-B$. As a result, we obtain (for details see [5]) a sequence of 4 maps with different normalized N/S ratio: for WMAP 1-year maps the $\mathrm{S} / \mathrm{N}$ yields $(0.5 ; 0.56 ; 1.0 ; 1.71)$, respectively, and smaller by a factor $\sqrt{3}$ for 3 -year ones. So, now we get maps based on WMAP 3-year data, in addition to those of WMAP 1-year ones, with 
different noise levels. The ellipticities in each of them have been estimated and are given in Fig. 6. We see that the behavior shown in Fig. 5 of [5] is confirmed and continued for smaller noise-to signal values, namely, the noise is affecting more strongly the smaller spots, along with clear monotonic convergence of ellipticities for all three pixel count spots.

Could one confirm this monotonic behavior of the noise in the simulated maps? To see this, we simulated CMB maps corresponding to the WMAP power spectrum without noise and then with WMAP's 3-year noise level. The simulations of the maps have been preformed by standard procedure. First, the Healpix [27] software was used to produce the signal no-noise maps corresponding the power spectrum for the cosmological parameters of WMAP3 data. Then, the noise was superposed using WMAP available $w$ band values for $\sigma$ (the average for the band, as well as for each four $w_{i}$, separately), weighted by $N_{o b s}$, the effective number of measurements (for details see http://lambda.gsfc.nasa.gov). The results for 50 simulated maps are given in Fig. 7. First, we see that, without noise the ellipticity is increasing for larger spots, which is contrary to the behavior in WMAP maps, as shown in Fig. 6. Second, the role of the noise is opposite for more than 20 and 50,100-pixel spots, which is strongly contradicting the monotonic behavior in real maps (Fig.6). Moreover, even for 20-pixel spots when simulated maps show the noise increasing the ellipticity as in real maps, numerically it implies no noise ellipticity about 2 , which is inconsistent with Fig. 6; with high confidence level of incompatibility, up to $10 \sigma$, in the values of the ellipticities.

Thus, the prediction in [5] that, both independence or strong dependence of the results on the noise in the simulated maps can be equally misleading, is confirmed. We now found, that the behavior of the noise level dependence is, moreover, not compatible with properties of the real maps.

WMAP 3-year data confirm the ellipticity around 2 in the CMB sky, found before for COBE, Boomerang and WMAP's 1-year maps.

In addition, the low noise level of WMAP 3-year data enable to conclude that, the ellipticity is a property not described by the cosmological model fitting the power spectrum of CMB. This is shown via the revealed incompatibility in the behavior of the noise, as well as in the dependence of the ellipticity of the size of the spots in the WMAP's and simulated maps. Namely, for WMAP's data a clear monotonic behavior of the noise in the ellipticity is shown. While in the simulated maps, for more than 50 and 100 pixel spots the role of the noise is contrary to that in the real maps. For more than 20-pixel spots, even though the simulated behavior is compatible with real data, numerically there is $10 \sigma$ discrepancy between the simulated 
and measured ellipticities. Finally, simulated maps violate the monotonic noise behavior in the real data.

If the ellipticity is due to geodesic mixing (with properties [19] fully corresponding to the measured ones) or to another large scale effect, then the interesting question is, how it is linked with the low quadrupole of the CMB [28, as well as with the dark energy, if the latter is due to relevant vacuum fluctuations defined by the boundary conditions of the Universe 30, 31, 32.

We are thankful to P. de Bernardis and his team for help and numerous discussions; G.Polenta is particularly thanked for help with simulations of CMB maps.

\section{References}

[1] de Bernardis P., et al, 2000, Nature, 404, 955.

[2] de Bernardis P., et al, 2003, IAU Symposium 216: Maps of the Cosmos, ASP Conf. Series; astro-ph/0311396.

[3] Gurzadyan V.G., Ade P.A.R., de Bernardis P. et al, 2003, Int.J.Mod.Phys. D12, 1859.

[4] Bennett C.L., et al., 2003, ApJS 148, 1.

[5] Gurzadyan V.G., de Bernardis P. et al, 2005, Mod.Phys.Lett. A20, 893; 2003, Nuovo Cimento, B118,1101.

[6] Gurzadyan V.G., Torres S., 1997, A \& A, 321, 19.

[7] Gurzadyan V.G., Ade P.A.R., de Bernardis P. et al, 2005, Mod.Phys.Lett., A20, 491.

[8] Eriksen H.K. et al, 2004, ApJ, 605, 14.

[9] Copi C.J., Huterer D., Starkman G.D., 2004, Phys. Rev. 70, 043515

[10] Schwarz D.J., et al, 2004, Phys.Rev.Lett. 93, 221301

[11] Land K., Magueijo J., 2005, Phys. Rev. D72, 101302.

[12] Copi C.J., Huterer D., Schwarz D.J., Starkman G.D., 2006, Mon.Not.Roy.Astron.Soc. 367, 79 
[13] Magueijo J., Sorkin R.D., 2006, astro-ph/0604410

[14] Cruz M., Cayon L., Martinez-Gonzalez E., et al, 2006, astro-ph/0603859

[15] McEwen J. D., Hobson M.P., Lasenby A.N., Mortlock D.J., 2006, astro-ph/0604305

[16] Spergel D. et al, 2006, astro-ph/0603449

[17] Page L. et al, 2006, astro-ph/0603450

[18] Hinshaw G. et al, 2006, astro-ph/0603451

[19] Gurzadyan V.G., Kocharyan A.A., 1992, A \& A, 260, 14; Europhys.Lett. 1993, 22, 231.

[20] Penrose R., The Road to Reality, Jonathan Cape, London, 2004.

[21] Anosov D.V., 1967, Comm. Steklov Mathematical Inst., vol.90.

[22] Gurzadyan V.G., 1999, Europhys.Lett., 46, 114.

[23] Bond J.R., Efstathiou G., 1987, MNRAS, 226, 655.

[24] Efstathiou G., 2003, MNRAS, 343, L95.

[25] Luminet J.-P. et al, 2003, Nature, 425, 593.

[26] Aurich R., Lustig S., Steiner F., Then H., 2005, Phys.Rev.Lett. 94, 021301.

[27] Górski, K.M., Hivon, E. and Wandelt, B.D., 1998, astro-ph/9812350 http://www.eso.org/kgorski/healpix/

[28] Copi C.J., Huterer D., Schwarz D.J., Starkman G.D., 2006, astro-ph/0605135

[29] Draine B.T., Lazarian A. 1998, ApJ, 494, L19.

[30] Gurzadyan V.G., Xue S.S. 2003, Mod.Phys.Lett. A18, 561.

[31] Vereshchagin G.V., Yegorian G., 2006, Phys.Lett. B636, 150; Class. Quant.Grav. 23, 5049; astro-ph/0610197.

[32] Djorgovski S.G., Gurzadyan V.G., 2006, Nucl. Phys. B (in press); astro-ph/0610204. 


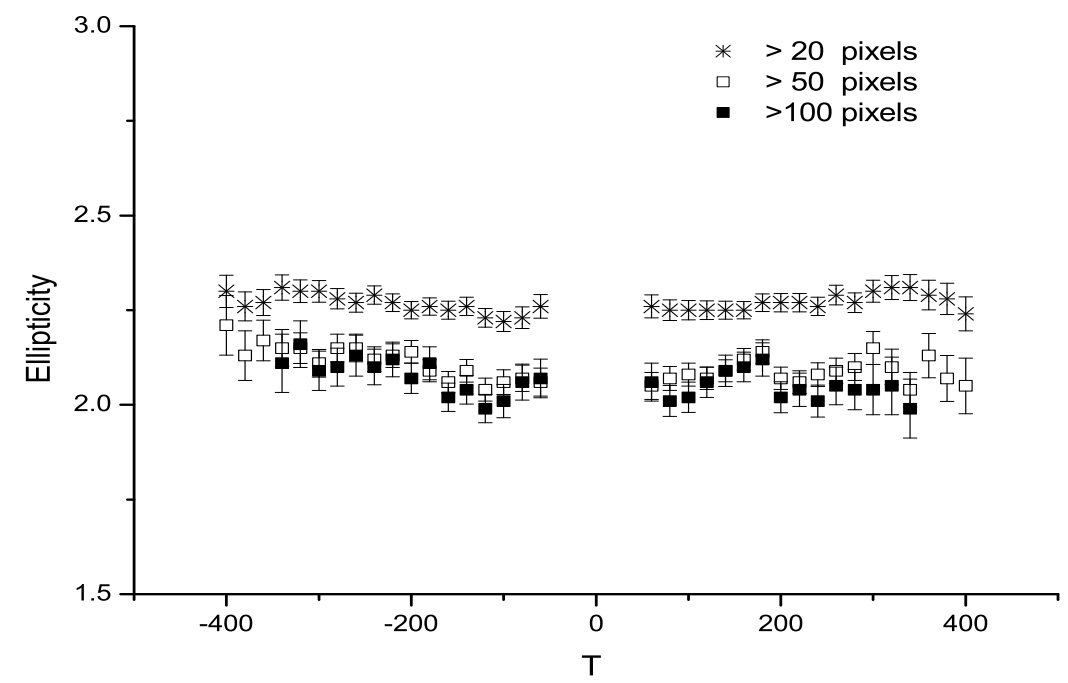

Figure 1: Ellipticity of anisotropies vs temperature threshold (in $\mu K$ ) for spots containing more than 20, 50 and 100 pixels in WMAP 3-year $94 \mathrm{GHz}$ sum map $A=(\mathrm{w} 1+\mathrm{w} 2) / 2$ and $\mathrm{B}=(\mathrm{w} 3+\mathrm{w} 4) / 2$. The error bars are statistical only. 


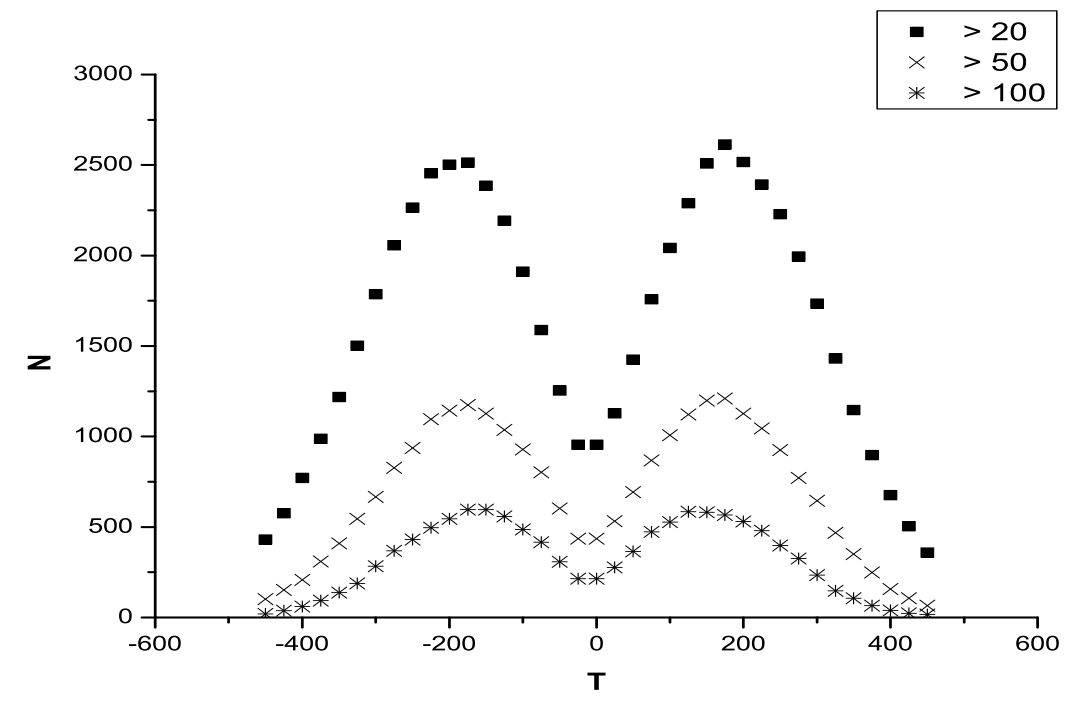

Figure 2: The number of anisotropy spots containing more than 20, 50 and 100 pixels vs the temperature threshold; cf. with WMAP 1-year data in 5] 


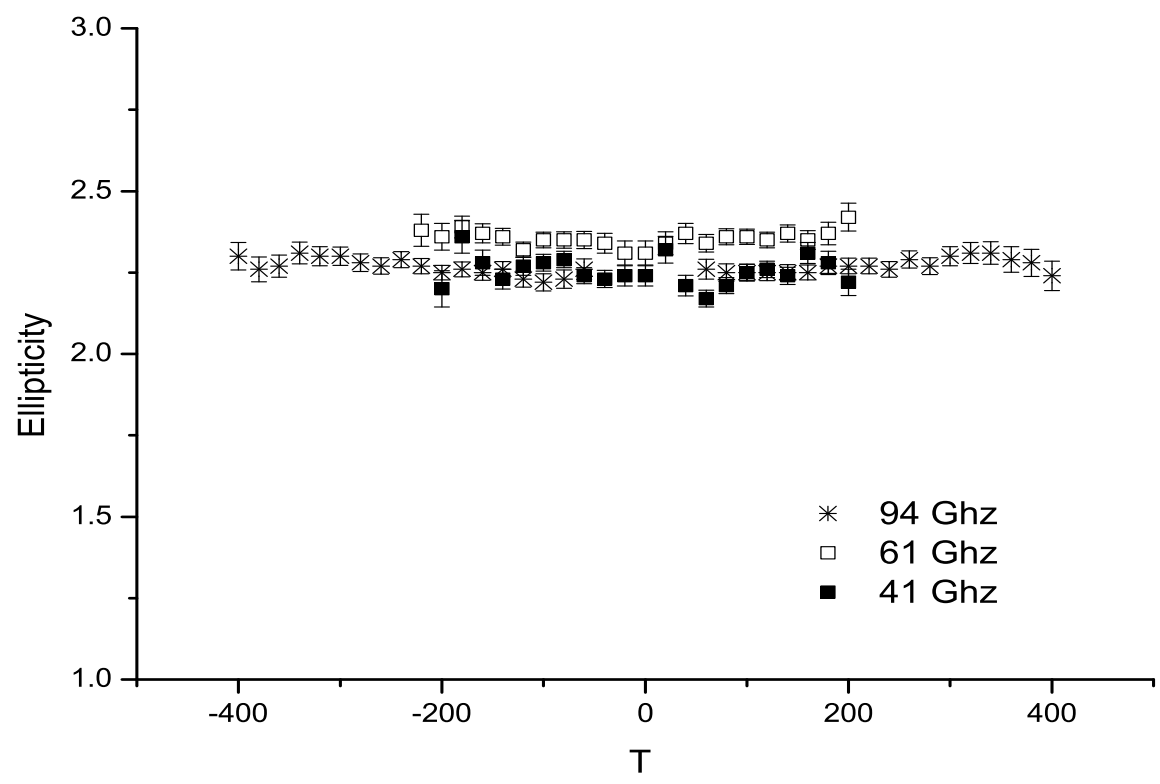

Figure 3: Band dependence of the ellipticity; this and Figs.4,5 are for more than 20 pixel spots. 


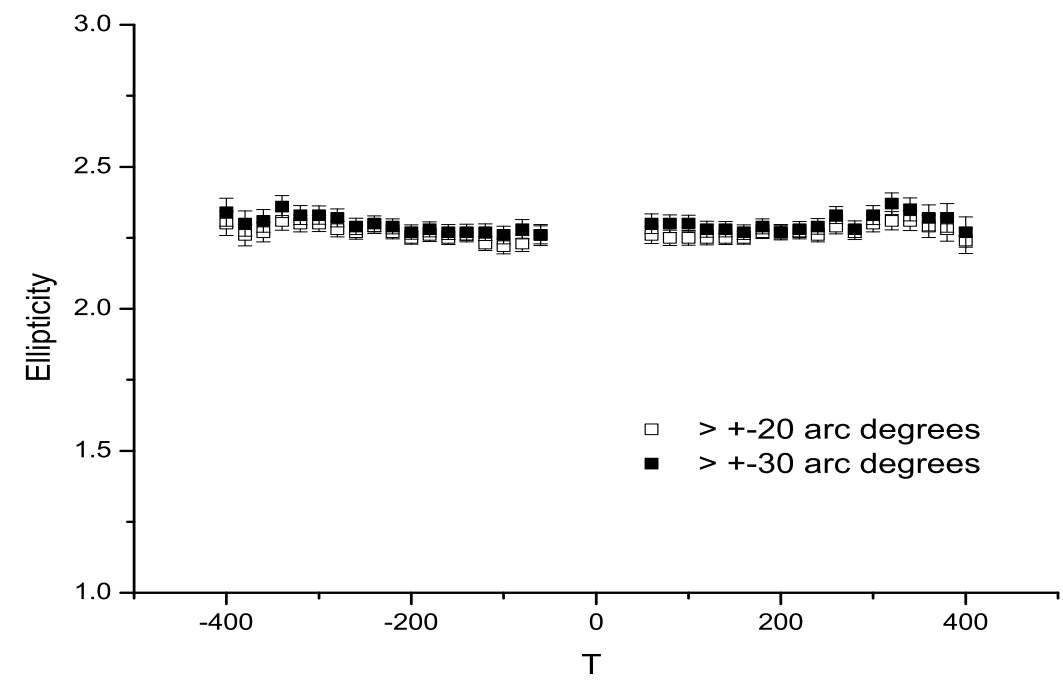

Figure 4: Ellipticity when $|b|<20^{\circ}$ and $|b|<30^{\circ}$ pixels are excluded. 


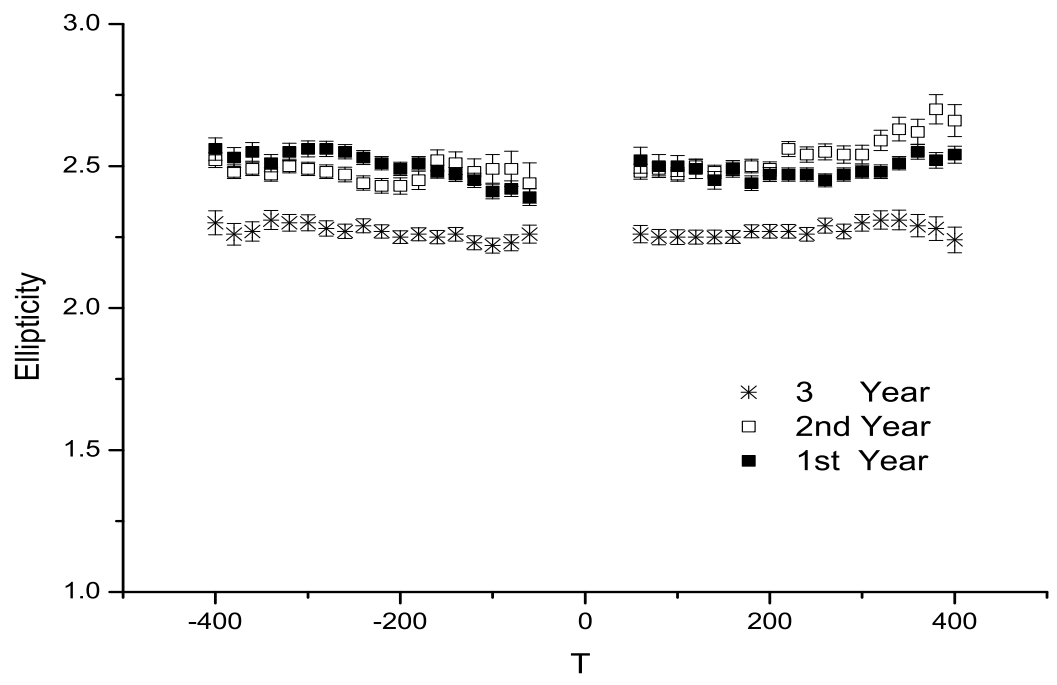

Figure 5: Ellipticity in 1st, 2nd yearly, and 3-year (i.e. the sum of three years) maps. 


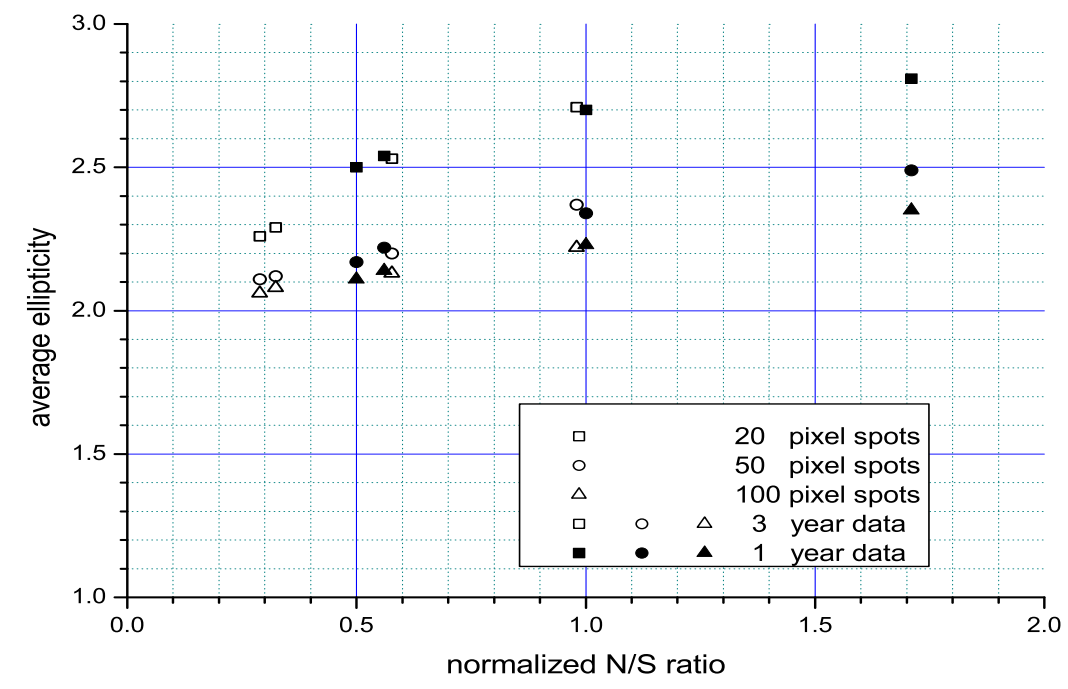

Figure 6: Average ellipticity vs noise-to-signal ratio for WMAP 1-year and 3 -year maps. The noise-to-signal ratio is normalized to 1 for 1 -year map from detector $w_{1}$ alone. 


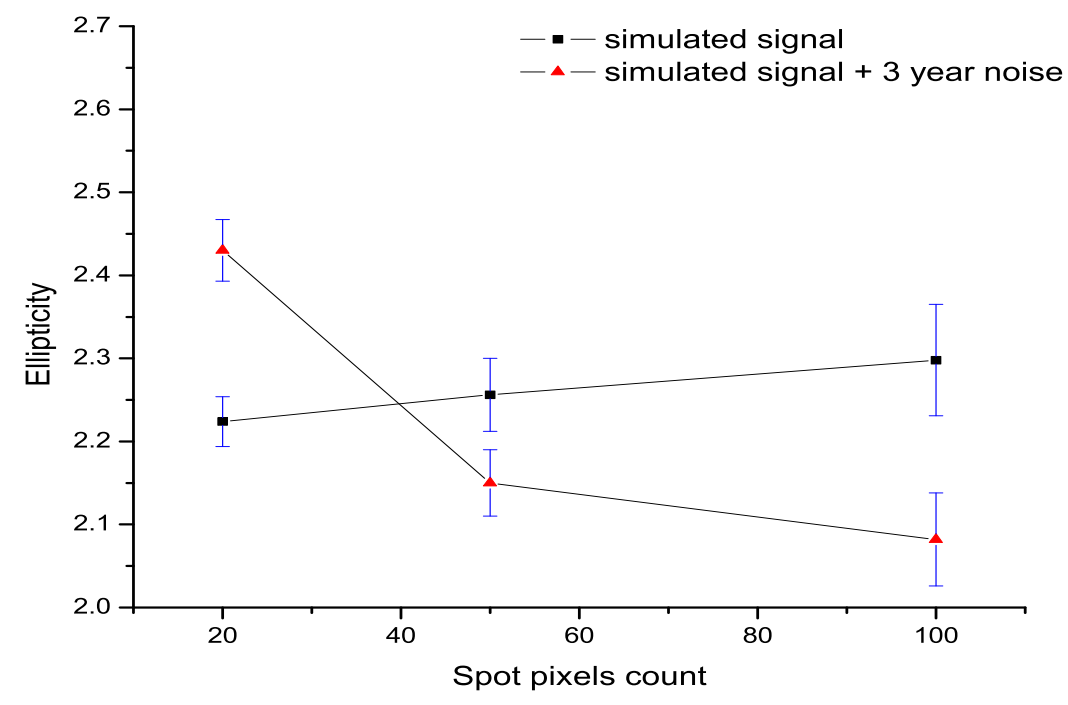

Figure 7: Average ellipticity in $50 \mathrm{CMB}$ maps simulated for the power spectrum parameters with and without noise. The inconsistency with Fig.6 is visible already in the non-monotonic behavior of the noise for spots with various pixel counts. 